\title{
ON NEW INEQUALITIES FOR $h$-CONVEX FUNCTIONS VIA RIEMANN-LIOUVILLE FRACTIONAL INTEGRATION
}

\author{
MEVLÜT TUNÇ
}

\begin{abstract}
In this paper, some new inequalities of the Hermite-Hadamard type for $h$-convex functions via Riemann-Liouville fractional integral are given.
\end{abstract}

\section{INTRODUCTION}

Let $f: I \subseteq \mathbb{R} \rightarrow \mathbb{R}$ be a convex function and let $a, b \in I$, with $a<b$. The following inequality;

$$
f\left(\frac{a+b}{2}\right) \leq \frac{1}{b-a} \int_{a}^{b} f(x) d x \leq \frac{f(a)+f(b)}{2}
$$

is known in the literature as Hadamard's inequality. Both inequalities hold in the reversed direction if $f$ is concave.

In [1], Varošanec introduced the following class of functions.

Definition 1. Let $h: J \subset \mathbb{R} \rightarrow \mathbb{R}$ be a positive function. We say that $f: I \subset \mathbb{R} \rightarrow$ $\mathbb{R}$ is $h$-convex function or that $f$ belongs to the class $S X(h, I)$, if $f$ is nonnegative and for all $x, y \in I$ and $\lambda \in(0,1)$ we have

$$
f(\lambda x+(1-\lambda) y) \leq h(\lambda) f(x)+h(1-\lambda) f(y) .
$$

If the inequality in (1.2) is reversed, then $f$ is said to be $h$-concave, i.e., $f \in$ $S V(h, I)$.

Obviously, if $h(\lambda)=\lambda$, then all nonnegative convex functions belong to $S X(h, I)$ and all nonnegative concave functions belong to $S V(h, I)$; if $h(\lambda)=\frac{1}{\lambda}$, then $S X(h, I)=$ $Q(I)$; if $h(\lambda)=1$, then $S X(h, I) \supseteq P(I)$ and if $h(\lambda)=\lambda^{s}$, where $s \in(0,1)$, then $S X(h, I) \supseteq K_{s}^{2}$. For some recent results for $h$-convex functions we refer to the interested reader to the papers [3]-[6].

Definition 2. [See [2] A function $h: J \rightarrow \mathbb{R}$ is said to be a superadditive function if

$$
h(x+y) \geq h(x)+h(y)
$$

for all $x, y \in J$.

In 3, Sarıkaya et al. proved the following Hadamard type inequalities for $h$-convex functions.

2000 Mathematics Subject Classification. 26D15, 41A51, 26 D10.

Key words and phrases. Riemann-Liouville fractional integral, $h$-convex function, Hadamard's inequality. 
Theorem 1. Let $f \in S X(h, I), a, b \in I$ with $a<b$ and $f \in L_{1}[a, b]$. Then

$$
\frac{1}{2 h\left(\frac{1}{2}\right)} f\left(\frac{a+b}{2}\right) \leq \frac{1}{b-a} \int_{a}^{b} f(x) d x \leq[f(a)+f(b)] \int_{0}^{1} h(\alpha) d \alpha .
$$

In [16, Sarıkaya et al. proved the following Hadamard type inequalities for fractional integrals as follows.

Theorem 2. Let $f:[a, b] \rightarrow \mathbb{R}$ be positive function with $0 \leq a<b$ and $f \in$ $L_{1}[a, b]$. If $f$ is convex function on $[a, b]$, then the following inequalities for fractional integrals hold:

$$
f\left(\frac{a+b}{2}\right) \leq \frac{\Gamma(\alpha+1)}{2(b-a)^{\alpha}}\left[J_{a^{+}}^{\alpha}(b)+J_{b^{-}}^{\alpha}(a)\right] \leq \frac{f(a)+f(b)}{2}
$$

with $\alpha>0$.

Now we give some necessary definitions and mathematical preliminaries of fractional calculus theory which are used throughout this paper.

Definition 3. Let $f \in L_{1}[a, b]$. The Riemann-Liouville integrals $J_{a^{+}}^{\alpha} f$ and $J_{b^{-}}^{\alpha} f$ of order $\alpha>0$ with $a \geq 0$ are defined by

$$
J_{a^{+}}^{\alpha} f(x)=\frac{1}{\Gamma(\alpha)} \int_{a}^{x}(x-t)^{\alpha-1} f(t) d t, \quad x>a
$$

and

$$
J_{b^{-}}^{\alpha} f(x)=\frac{1}{\Gamma(\alpha)} \int_{x}^{b}(t-x)^{\alpha-1} f(t) d t, \quad x<b
$$

respectively where $\Gamma(\alpha)=\int_{0}^{\infty} e^{-u} u^{\alpha-1} d u$. Here is $J_{a^{+}}^{0} f(x)=J_{b^{-}}^{0} f(x)=f(x)$.

In the case of $\alpha=1$, the fractional integral reduces to the classical integral.

For some recent results connected with fractional integral inequalities see [8]-[15] and [16.

In [16, Sarikaya et al. proved a variant of the identity is established by Dragomir and Agarwal in [7, Lemma 2.1] for fractional integrals as the following.

Lemma 1. Let $f:[a, b] \rightarrow \mathbb{R}$ be a differentiable mapping on $(a, b)$ with $a<b$. If $f^{\prime} \in L[a, b]$, then the following equality for fractional integrals holds:

$$
\begin{aligned}
& \frac{f(a)+f(b)}{2}-\frac{\Gamma(\alpha+1)}{2(b-a)^{\alpha}}\left[J_{a^{+}}^{\alpha} f(b)+J_{b^{-}}^{\alpha} f(a)\right] \\
= & \frac{b-a}{2} \int_{0}^{1}\left[(1-t)^{\alpha}-t^{\alpha}\right] f^{\prime}(t a+(1-t) b) d t .
\end{aligned}
$$

The aim of this paper is to establish Hadamard type inequalities for $h$-convex functions via Riemann-Liouville fractional integral. 


\section{MAIN RESULTS}

Theorem 3. Let $f \in S X(h, I), a, b \in I$ with $a<b$ and $f \in L_{1}[a, b]$. Then one has inequality for $h$-convex functions via fractional integrals

$$
\begin{aligned}
& \frac{\Gamma(\alpha)}{(b-a)^{\alpha}}\left[J_{a^{+}}^{\alpha}(b)+J_{b^{-}}^{\alpha}(a)\right] \\
\leq & {[f(a)+f(b)] \int_{0}^{1} t^{\alpha-1}[h(t)+h(1-t)] d t } \\
\leq & \frac{2[f(a)+f(b)]}{(\alpha p-p+1)^{\frac{1}{p}}}\left(\int_{0}^{1}(h(t))^{q} d t\right)^{\frac{1}{q}}
\end{aligned}
$$

where $p^{-1}+q^{-1}=1$.

Proof. Since $f \in S X(h, I)$, we have

$$
f(t x+(1-t) y) \leq h(t) f(x)+h(1-t) f(y)
$$

and

$$
f((1-t) x+t y) \leq h(1-t) f(x)+h(t) f(y) .
$$

By adding these inequalities we get

$$
f(t x+(1-t) y)+f((1-t) x+t y) \leq[h(t)+h(1-t)][f(x)+f(y)] .
$$

By using (2.2) with $x=a$ and $y=b$ we have

$$
f(t a+(1-t) b)+f((1-t) a+t b) \leq[h(t)+h(1-t)][f(a)+f(b)] .
$$

Then multiplying both sides of (2.3) by $t^{\alpha-1}$ and integrating the resulting inequality with respect to $t$ over $[0,1]$, we get

$$
\begin{aligned}
& \int_{0}^{1} t^{\alpha-1}[f(t a+(1-t) b)+f((1-t) a+t b)] d t \\
\leq & \int_{0}^{1} t^{\alpha-1}[h(t)+h(1-t)][f(a)+f(b)] d t \\
\leq & {[f(a)+f(b)] \int_{0}^{1} t^{\alpha-1}[h(t)+h(1-t)] d t } \\
& \frac{\Gamma(\alpha)}{(b-a)^{\alpha}}\left[J_{a^{+}}^{\alpha}(b)+J_{b^{-}}^{\alpha}(a)\right]
\end{aligned}
$$

and thus the first inequality is proved. 
To obtain the second inequality in (2.1), by using Hölder inequality for the right hand side of (2.4), we obtain

$$
\begin{aligned}
& \int_{0}^{1} t^{\alpha-1}[h(t)+h(1-t)] d t \\
\leq & \left(\int_{0}^{1}\left(t^{\alpha-1}\right)^{p} d t\right)^{\frac{1}{p}}\left(\int_{0}^{1}(h(t)+h(1-t))^{q} d t\right)^{\frac{1}{q}} \\
= & \left(\left.\frac{t^{\alpha p-p+1}}{\alpha p-p+1}\right|_{0} ^{1}\right)^{\frac{1}{p}}\left(\int_{0}^{1}(h(t)+h(1-t))^{q} d t\right)^{\frac{1}{q}} \\
= & \left(\frac{1}{\alpha p-p+1}\right)^{\frac{1}{p}}\left(\int_{0}^{1}(h(t)+h(1-t))^{q} d t\right)^{\frac{1}{q}}
\end{aligned}
$$

Then using Minkowski inequality

$$
\begin{aligned}
& \left(\frac{1}{\alpha p-p+1}\right)^{\frac{1}{p}}\left(\int_{0}^{1}(h(t)+h(1-t))^{q} d t\right)^{\frac{1}{q}} \\
\leq & \left(\frac{1}{\alpha p-p+1}\right)^{\frac{1}{p}}\left[\left(\int_{0}^{1}(h(t))^{q} d t\right)^{\frac{1}{q}}+\left(\int_{0}^{1}(h(1-t))^{q} d t\right)^{\frac{1}{q}}\right] \\
= & \frac{2}{(\alpha p-p+1)^{\frac{1}{p}}}\left(\int_{0}^{1}(h(t))^{q} d t\right)^{\frac{1}{q}}
\end{aligned}
$$

where the proof is completed.

Remark 1. If we choose $\alpha=1$ in Theorem 1, we obtain

$$
\begin{aligned}
\frac{1}{b-a} \int_{a}^{b} f(x) d x & \leq[f(a)+f(b)] \int_{0}^{1} h(t) d t \\
& \leq[f(a)+f(b)]\left(\int_{0}^{1}(h(t))^{q} d t\right)^{\frac{1}{q}} .
\end{aligned}
$$

Corollary 1. (1) If we choose $h(\lambda)=\lambda$ in Remark [1, we get

$$
\frac{1}{b-a} \int_{a}^{b} f(x) d x \leq \frac{f(a)+f(b)}{2}=\frac{f(a)+f(b)}{(q+1)^{\frac{1}{q}}}
$$

for ordinary convex functions.

(2) If we choose $h(\lambda)=1$ in Remark 1, we get

$$
\frac{2}{b-a} \int_{a}^{b} f(x) d x \leq 2(f(a)+f(b))
$$

for P-functions. This inequality is refinement of right hand side of (1.1) for $P$-functions.

(3) If we choose $h(\lambda)=\lambda^{s}$ in Remark 1, we get

$$
\frac{1}{b-a} \int_{0}^{1} f(x) d x \leq \frac{f(a)+f(b)}{s+1} \leq \frac{f(a)+f(b)}{(s q+1)^{\frac{1}{q}}}
$$

for $s$-convex functions in the second sense with $s \in(0,1]$. 
Theorem 4. Let $f \in S X(h, I), a, b \in I$ with $a<b, h$ is superadditive on $I$ and $f \in L_{1}[a, b], h \in L_{1}[0,1]$. Then one has inequality for $h$-convex functions via fractional integrals

$$
\frac{\Gamma(\alpha)}{(b-a)^{\alpha}}\left[J_{a^{+}}^{\alpha}(b)+J_{b^{-}}^{\alpha}(a)\right] \leq \frac{h(1)}{\alpha}[f(a)+f(b)] .
$$

Proof. Since $f \in S X(h, I)$, we have

$$
f(t x+(1-t) y) \leq h(t) f(x)+h(1-t) f(y)
$$

and

$$
f((1-t) x+t y) \leq h(1-t) f(x)+h(t) f(y) .
$$

By adding these inequalities we get

$$
f(t x+(1-t) y)+f((1-t) x+t y) \leq[h(t)+h(1-t)][f(x)+f(y)] .
$$

By using (2.6) with $x=a$ and $y=b$ and $h$ is superadditive, we get

$$
f(t a+(1-t) b)+f((1-t) a+t b) \leq h(1)[f(a)+f(b)] .
$$

Then multiplying both sides of (2.7) by $t^{\alpha-1}$ and integrating the resulting inequality with respect to $t$ over $[0,1]$, we get

$$
\begin{gathered}
\int_{0}^{1} t^{\alpha-1}[f(t a+(1-t) b)+f((1-t) a+t b)] d t \\
\leq \int_{0}^{1} t^{\alpha-1} h(1)[f(a)+f(b)] d t \\
\frac{\Gamma(\alpha)}{(b-a)^{\alpha}}\left[J_{a^{+}}^{\alpha}(b)+J_{b^{-}}^{\alpha}(a)\right] \\
\leq h(1)[f(a)+f(b)] \int_{0}^{1} t^{\alpha-1} d t .
\end{gathered}
$$

This completes the proof.

Remark 2. If we choose $\alpha=1$ in Theorem 4, then (2.5) reduce to special version of right hand side of (1.4).

Theorem 5. Let $h: J \subset \mathbb{R} \rightarrow \mathbb{R}$ and $f:[a, b] \rightarrow \mathbb{R}$ be positive functions with $0 \leq a<b$ and $h^{q} \in L_{1}[0,1], f \in L_{1}[a, b]$. If $\left|f^{\prime}\right|$ is an $h$-convex mapping on $[a, b]$, then the following inequality for fractional integrals holds,

$$
\begin{aligned}
& \left|\frac{f(a)+f(b)}{2}-\frac{\Gamma(\alpha+1)}{2(b-a)^{\alpha}}\left[J_{a+}^{\alpha} f(b)+J_{b-}^{\alpha} f(a)\right]\right| \\
\leq & \frac{(b-a)\left[\left|f^{\prime}(a)\right|+\left|f^{\prime}(b)\right|\right]}{2}\left[\left(\frac{2^{\alpha p+1}-1}{2^{\alpha p+1}(\alpha p+1)}\right)^{\frac{1}{p}}-\left(\frac{1}{2^{\alpha p+1}(\alpha p+1)}\right)^{\frac{1}{p}}\right] \\
\times & {\left[\left(\int_{0}^{\frac{1}{2}}(h(t))^{q} d t\right)^{\frac{1}{q}}+\left(\int_{\frac{1}{2}}^{1}(h(t))^{q} d t\right)^{\frac{1}{q}}\right] }
\end{aligned}
$$

where $\alpha>0, p>1$ and $p^{-1}+q^{-1}=1$. 
Proof. From Lemma 1 and using the properties of modulus, we have

$$
\begin{aligned}
& \left|\frac{f(a)+f(b)}{2}-\frac{\Gamma(\alpha+1)}{2(b-a)^{\alpha}}\left[J_{a+}^{\alpha} f(b)+J_{b-}^{\alpha} f(a)\right]\right| \\
\leq & \frac{b-a}{2} \int_{0}^{1}\left|(1-t)^{\alpha}-t^{\alpha}\right|\left|f^{\prime}(t a+(1-t) b)\right| d t .
\end{aligned}
$$

Since $\left|f^{\prime}\right|$ is $h$-convex on $[a, b]$, we have

$$
\begin{aligned}
& \left|\frac{f(a)+f(b)}{2}-\frac{\Gamma(\alpha+1)}{2(b-a)^{\alpha}}\left[J_{a+}^{\alpha}(b)+J_{b-}^{\alpha}(a)\right]\right| \\
\leq & \frac{b-a}{2}\left\{\int_{0}^{\frac{1}{2}}\left[(1-t)^{\alpha}-t^{\alpha}\right]\left[h(t)\left|f^{\prime}(a)\right|+h(1-t)\left|f^{\prime}(b)\right|\right] d t\right. \\
& \left.+\int_{\frac{1}{2}}^{1}\left[t^{\alpha}-(1-t)^{\alpha}\right]\left[h(t)\left|f^{\prime}(a)\right|+h(1-t)\left|f^{\prime}(b)\right|\right] d t\right\} \\
= & \frac{b-a}{2}\left\{\left|f^{\prime}(a)\right| \int_{0}^{\frac{1}{2}}(1-t)^{\alpha} h(t) d t-\left|f^{\prime}(a)\right| \int_{0}^{\frac{1}{2}} t^{\alpha} h(t) d t\right. \\
& +\left|f^{\prime}(b)\right| \int_{0}^{\frac{1}{2}}(1-t)^{\alpha} h(1-t) d t-\left|f^{\prime}(b)\right| \int_{0}^{\frac{1}{2}} t^{\alpha} h(1-t) d t \\
& +\left|f^{\prime}(a)\right| \int_{\frac{1}{2}}^{1} t^{\alpha} h(t) d t-\left|f^{\prime}(a)\right| \int_{\frac{1}{2}}^{1}(1-t)^{\alpha} h(t) d t \\
& \left.+\left|f^{\prime}(b)\right| \int_{\frac{1}{2}}^{1} t^{\alpha} h(1-t) d t-\left|f^{\prime}(b)\right| \int_{\frac{1}{2}}^{1}(1-t)^{\alpha} h(1-t) d t\right\} .
\end{aligned}
$$

In the right hand side of above inequality by using Hölder inequality for $p^{-1}+q^{-1}=$ 1 and $p>1$, we get

$$
\begin{aligned}
& \int_{0}^{\frac{1}{2}}(1-t)^{\alpha} h(t) d t=\int_{\frac{1}{2}}^{1} t^{\alpha} h(1-t) d t \leq\left[\frac{2^{\alpha p+1}-1}{2^{\alpha p+1}(\alpha p+1)}\right]^{\frac{1}{p}}\left(\int_{0}^{\frac{1}{2}}[h(t)]^{q} d t\right)^{\frac{1}{q}}, \\
& \int_{0}^{\frac{1}{2}}(1-t)^{\alpha} h(1-t) d t=\int_{\frac{1}{2}}^{1} t^{\alpha} h(t) d t \leq\left[\frac{2^{\alpha p+1}-1}{2^{\alpha p+1}(\alpha p+1)}\right]^{\frac{1}{p}}\left(\int_{\frac{1}{2}}^{1}[h(t)]^{q} d t\right)^{\frac{1}{q}}, \\
& \int_{0}^{\frac{1}{2}} t^{\alpha} h(t) d t=\int_{\frac{1}{2}}^{1}(1-t)^{\alpha} h(1-t) d t \leq\left[\frac{1}{2^{\alpha p+1}(\alpha p+1)}\right]^{\frac{1}{p}}\left(\int_{0}^{\frac{1}{2}}[h(t)]^{q} d t\right)^{\frac{1}{q}}
\end{aligned}
$$

and

$$
\int_{0}^{\frac{1}{2}} t^{\alpha} h(1-t) d t=\int_{\frac{1}{2}}^{1}(1-t)^{\alpha} h(t) d t \leq\left[\frac{1}{2^{\alpha p+1}(\alpha p+1)}\right]^{\frac{1}{p}}\left(\int_{\frac{1}{2}}^{1}[h(t)]^{q} d t\right)^{\frac{1}{q}} .
$$


Then using the above inequalities in the right hand side of (2.9), we get

$$
\begin{aligned}
& \left|\frac{f(a)+f(b)}{2}-\frac{\Gamma(\alpha+1)}{2(b-a)^{\alpha}}\left[J_{a+}^{\alpha}(b)+J_{b-}^{\alpha}(a)\right]\right| \\
\leq & \frac{b-a}{2}\left\{\left|f^{\prime}(a)\right|\left\{\left(\left[\frac{2^{\alpha p+1}-1}{2^{\alpha p+1}(\alpha p+1)}\right]^{\frac{1}{p}}-\left[\frac{1}{2^{\alpha p+1}(\alpha p+1)}\right]^{\frac{1}{p}}\right)\left(\int_{0}^{\frac{1}{2}}[h(t)]^{q} d t\right)^{\frac{1}{q}}\right\}\right. \\
& \left.+\left(\left[\frac{2^{\alpha p+1}-1}{2^{\alpha p+1}(\alpha p+1)}\right]^{\frac{1}{p}}-\left[\frac{1}{2^{\alpha p+1}(\alpha p+1)}\right]^{\frac{1}{p}}\right)\left(\int_{\frac{1}{2}}^{1}[h(t)]^{q} d t\right)^{\frac{1}{q}}\right\} \\
& +\left|f^{\prime}(b)\right|\left\{\left(\left[\frac{2^{\alpha p+1}-1}{2^{\alpha p+1}(\alpha p+1)}\right]^{\frac{1}{p}}-\left[\frac{1}{2^{\alpha p+1}(\alpha p+1)}\right]^{\frac{1}{p}}\right)\left(\int_{\frac{1}{2}}^{1}[h(t)]^{q} d t\right)^{\frac{1}{q}}\right. \\
& \left.+\left(\left[\frac{2^{\alpha p+1}-1}{2^{\alpha p+1}(\alpha p+1)}\right]^{\frac{1}{p}}-\left[\frac{1}{2^{\alpha p+1}(\alpha p+1)}\right]^{\frac{1}{p}}\right)\left(\int_{0}^{\frac{1}{2}}[h(t)]^{q} d t\right)^{\frac{1}{q}}\right\} \\
= & \frac{b-a}{2}\left\{| f ^ { \prime } ( a ) | \left(\left[\frac{2^{\alpha p+1}-1}{2^{\alpha p+1}(\alpha p+1)}\right]^{\frac{1}{p}}-\left[\frac{1}{2^{\alpha p+1}(\alpha p+1)}\right]^{\frac{1}{p}}\left[\left(\int_{0}^{\frac{1}{2}}[h(t)]^{q} d t\right)^{\frac{1}{q}}+\left(\int_{\frac{1}{2}}^{1}[h(t)]^{q} d t\right)^{\frac{1}{q}}\right]\right.\right. \\
& \left.+\left|f^{\prime}(b)\right|\left(\left[\frac{2^{\alpha p+1}-1}{2^{\alpha p+1}(\alpha p+1)}\right]^{\frac{1}{p}}-\left[\frac{1}{2^{\alpha p+1}(\alpha p+1)}\right]^{\frac{1}{p}}\right)\left[\left(\int_{0}^{\frac{1}{2}}[h(t)]^{q} d t\right)^{\frac{1}{q}}+\left(\int_{\frac{1}{2}}^{1}[h(t)]^{q} d t\right)^{\frac{1}{q}}\right]\right\} \\
= & \frac{(b-a)\left[\left|f^{\prime}(a)\right|+\left|f^{\prime}(b)\right|\right]}{2}\left[\left(\frac{2^{\alpha p+1}-1}{2^{\alpha p+1}(\alpha p+1)}\right)^{\frac{1}{p}}-\left(\frac{1}{2^{\alpha p+1}(\alpha p+1)}\right)^{\frac{1}{p}}\right] \\
& \times\left[\left(\int_{0}^{\frac{1}{2}}(h(t))^{q} d t\right)^{\frac{1}{q}}+\left(\int_{\frac{1}{2}}^{1}(h(t))^{q} d t\right)\right.
\end{aligned}
$$

which is the desired result. The proof is completed.

\section{REFERENCES}

[1] S. Varošanec, On h-convexity, J. Math. Anal. Appl., 326 (2007), 303-311.

[2] H. Alzer, A superadditive property of Hadamard's gamma function, Abh. Math. Semin. Univ. Hambg., 79 (2009), 11-23.

[3] M. Z. Sarıkaya, A. Sağlam and H. Yıldırım, On some Hadamard-type inequalities for $h$-convex functions. Journal of Mathematical Inequalities, 2 (3) (2008), 335-341.

[4] M. Bombardelli and S. Varošanec, Properties of $h$-convex functions related to the HermiteHadamard-Fejér inequalities, Computers and Mathematics with Applications, 58 (2009), 1869-1877.

[5] M.Z. Sarıkaya, E. Set and M.E. Özdemir, On some new inequalities of Hadamard type involving $h$-convex functions, Acta Math. Univ. Comenianae, Vol. LXXIX, 2 (2010), pp. 265-272.

[6] P. Burai and A. Házy, On approximately $h$-convex functions, Journal of Convex Analysis, 18 (2) (2011).

[7] S.S. Dragomir and R. P. Agarwal, Two inequalities for differentiable mappings and applications to special means of real numbers and trapezoidal formula, Appl. Math. Lett., 11 (5) (1998), 91-95.

[8] S. Belarbi and Z. Dahmani, On some new fractional integral inequalities, J. Ineq. Pure and Appl. Math., 10(3) (2009), Art. 86. 
[9] Z. Dahmani, New inequalities in fractional integrals, International Journal of Nonlinear Science, 9(4) (2010), 493-497.

[10] M.E. Özdemir, H. Kavurmacı and M. Avcı, New inequalities of Ostrowski type for mappings whose derivatives are $(\alpha, m)$-convex via fractional integrals, RGMIA Research Report Collection, 15(2012), Article 10, 8 pp.

[11] M.E. Özdemir, H. Kavurmacı and Ç. Yıldız, Fractional integral inequalities via $s$-convex functions, arXiv:1201.4915 1 [math.CA] 24 Jan 2012.

[12] E. Set, New inequalities of Ostrowski type for mappings whose derivatives are $s$-convex in the second sense via fractional integrals, Comput. Math. Appl., In Press, Corrected Proof, 29 December 2011.

[13] Z. Dahmani, On Minkowski and Hermite-Hadamard integral inequalities via fractional integration, Ann. Funct. Anal. 1(1) (2010), 51-58.

[14] Z. Dahmani and L. Tabharit, S. Taf, Some fractional integral inequalities, Nonl. Sci. Lett. A., 1(2) (2010), 155-160.

[15] Z. Dahmani, L. Tabharit and S. Taf, New generalizations of Grüss inequality using RiemannLiouville fractional integrals, Bull. Math. Anal. Appl., 2(3) (2010), 93-99.

[16] M. Z. Sarıkaya, E. Set, H. Yaldiz and N. Başak, Hermite-Hadamard's inequalities for fractional integrals and related fractional inequalities, Mathematical and Computer Modelling, In Press.

University of Kilis 7 Aralik, Faculty of Science and Arts, Department of MatheMatics, 79000, Kilis, TURKey

E-mail address: mevluttunc@kilis.edu.tr 